\title{
IoT Based Human Intrusion Detection System using Lab View
}

\author{
Kalathiripi Rambabu, V.Haritha, S.Nikhil Srinivas, P.Sanjana Reddy
}

\begin{abstract}
Recent times there is an increase in thefts in the recent past. This creates a very bad environment for people to live in fear. The problem with home security in the modern world is a cause for concern. The conventional intruder detection system now we are using are highly expensive and there can be a possibility of false alarms. This problem is fixed by building a home intruder detection system that can accurately detect a human intruder, while filtering out movements that are caused due to any other moving objects using LabVIEW and Python. The images that were acquired and analyzed through frame comparisons are converted to gray scale images and then processed to detect an intruder. Here LabVIEW works as server and Python works as a client. At client video is acquired continuously, video is converted into images. Images are processed and information is send to the server. Server displays the status of the intruder with date and time. If the intruder is present then the system compares the intruder's data with the data in the system. The images that were acquired and analyzed through frame comparisons converted to gray scale images that represent change, and then filtered through a series of image refining VI's, helping to enhance our change detection results. If the data matches then the processing stops, if not then the system alerts the user through SMS or email if any intruder has been detected and sends the image to the user app. Through app we can make an alarm.
\end{abstract}

Keywords-LabVIEW, IOT, Raspberry pi.

\section{INTRODUCTION}

Intrusion, the act of someone that you don't know, who enters into your area without your permission, is on the rise. A human intrusion detection system is designed to detect an unauthorized entry into a building or a protected area and deny such unauthorized access to protect personnel and property from damage or harm. Security systems are mainly used in residential, commercial, industrial, and military properties for protection against burglary (theft) or property damage, as well as personal protection against intruders. The human presence of security guard may not be completely trustworthy. In such cases, this system provides proper detection of intruder and provides security. By using this system, we can reduce robbery by detecting the intruder. So we can respond quickly such that no harm takes place in our home.In the designed system, he camera is kept outside the room and the continuous video is captured by the camera. we designed our system in such a way that as soon as intruder enters the room, the processing takes place at the

Revised Manuscript Received on April 12, 2019.

Kalathiripi Rambabu, B.V.Raju Institute of Technology, Narsapur, Medak, Telangana. India.

V.Haritha, B.V.Raju Institute of Technology, Narsapur, Medak, Telangana. India

S.Nikhil Srinivas, B.V.Raju Institute of Technology, Narsapur, Medak, Telangana. India

P.Sanjana Reddy, B.V.Raju Institute of Technology, Narsapur, Medak, Telangana. India client. The message is obtained at the server by giving information of the time and date when intruder enters.

\section{PROBLEM STATEMENT}

Theft and Robbery are some of the dangerous threats across the globe in recent times. The home security systems have been constantly failing to safeguard houses and offices.

According to a survey conducted by FBI, all the intrusion problems are being occurred in absence of the owners in the home. In the year 2012, a house is being invaded by the intruders for every twenty seconds. Among these, only $10 \%$ of them end up in arrests, and rest of them are still suffering from intruders. There are many solutions for this problem like home automation systems, alarm detection systems etc. but there is a chance of producing false alarms by these Systems.

This is because, generally in home, along with human beings there may be pet animals too. These detection systems when come across these pets, produce false alarms thinking them to be intruders. This is the main reason for those systems to be failed. Thus, there is a requirement of a system that can differentiate those pet animals form human beings and then detect the intruder who entered the home or office. There are several intruder detection systems which can differentiate humans from pet animals. But cost is the main problem for those systems as their maintenance is high. The proposed is system very efficient in all aspects including the cost.

\section{EXISTING SYSTEMS}

Now a days, there are many existing intruder detection systems for surveillance and security systems. They either need human intervention to detect intruder or need a long work for the installation and there is also a possibility of false alarms. In Surveillance system, the intruder is detected using the video recordable cameras which are already installed and stored in an external storage disks. But in this system needs a huge investment for installing, storing and monitoring the activities. Thought the occurrence of activity is less, the footage is to be deleted, after examining by the owner. This leads to waste of man hour as he should watch the complete video and this may lead to missing of small details during analysis. The second system is radar-based human intruder detection system.

Which uses radar to detect human intruder by transmitting pulses of radio waves or microwaves that will bounce off any object in their path. These systems are easily available and cost effective but it causes radiation as radio signals are 
used. The other type is burglar alarm systems which are electronic alarms designed for alerting the owner when intruder enters. These are divided into several types, such as passive IR (PIR) motion detector system, ultrasonic motion detector system, glass-break detector system, photoelectric beam system, vibration sensor system. Eventhough, they do not require human support for detecting intruder, there is a possibility of false alarm. This may lead tounnecessary pressure to the owner.

\section{PROPOSED SYSTEMS}

In proposed system, a continuous video is taken by the camera to detect an intruder and to differentiate him with the moving objects by using face recognition API in python. If the person is detected, the acquired image is compared with the images in the database which avoids false alarms. If he is not present in the database, it sends message and image of the intruder to the owner through mail. The owner should give the acknowledgement through app if he is really an intruder. As soon as he gives acknowledgement, alarm is generated.

\section{A. Working Model Of Proposed System:-}

The system consists of a Raspberry Pi, to which two cameras are connected, one is for acquiring video continuously and other is for taking a snapshot of intruder. The system consists of a server and a client. LabVIEW works as a server, which shows the status of intruder. Python works as a client, which continuously monitors the status of intruder. The camera continuously monitors the area and acquires the video. From the acquired video, images are taken for every period of time. The obtained images are converted into RGB images. These images are further processed, to find out whether intruder is present or not. The processing stops in the case if intruder is not present. If the intruder is present, the system differentiates between pets and human beings. If intruder is a pet or an animal, processing stops. If not, the information is send to the server. The server displays the status of intruder with date and time. And further, the processed image is compared with the database in the system. If the person's image matches with the images in the storage of the system, the processing terminates. If not the user gets a mail and a message to the developed app which has status of the intruder's presence and the image of the intruder. From the app the user controls the system by giving an alarm from any place which helps to alert the people.

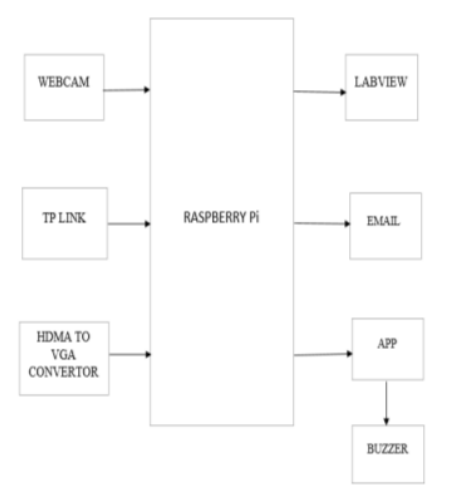

Fig 1: Block Diagram

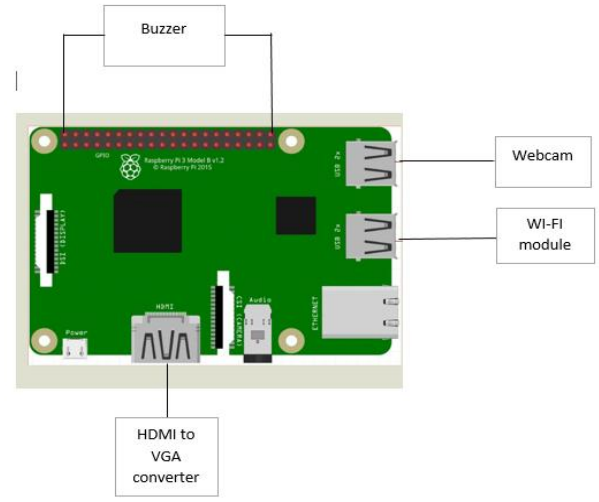

Fig 2: Schematic Diagram

\section{B. Raspberry Pi:-}

In proposed intruder detection system, Raspberry pi is used as a micro controller. The Raspberry Pi is a credit-card sized computer that plugs into a computer monitor or TV, and uses a standard keyboard and mouse and used in place of CPU. It is low in cost compared to CPU. It is mainly used for students for learning programming languages like python and scratch. In this project, we use Raspberry Pi 3 model B v1.2 which is a third generation model. Raspberry Pi $\mathbf{3}$ is a development board in PI series and can be considered as a single board computer that works on LINUX operating system. The board consists of many features and also has a very good processing speed which is suitable for advanced applications. The Raspberry Pi is a open-source platform that has a thriving community of its own, which is similar to that of the Arduino.

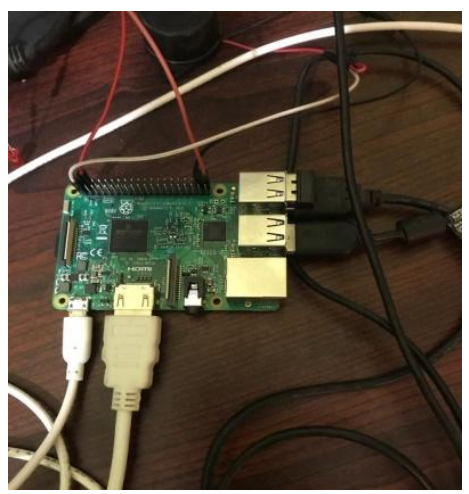

Fig 3: Raspberry Pi

In this project, two cameras are used which are connected to raspberry pi for intruder detection. One of the camera is used for taking the continuous video and other is for taking photos of the intruder. The ADNET HDMI to VGA cable is used for connecting the raspberry pi to the monitor or TV. The HDMI port is connected to raspberry pi and VGA cable is connected to TV or monitor.

The sdcard is inserted into one of the port of Raspberry pi which consists of python programming for face recognition, face comparison and for generating alarm. A buzzer and LED are connected to the raspberry pi.

\section{Python-Flow Chart:-}

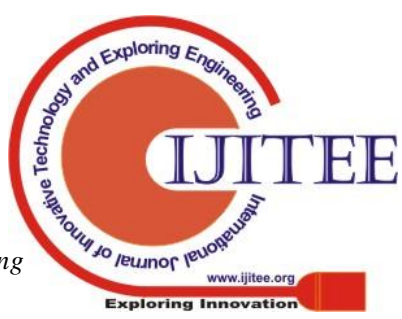




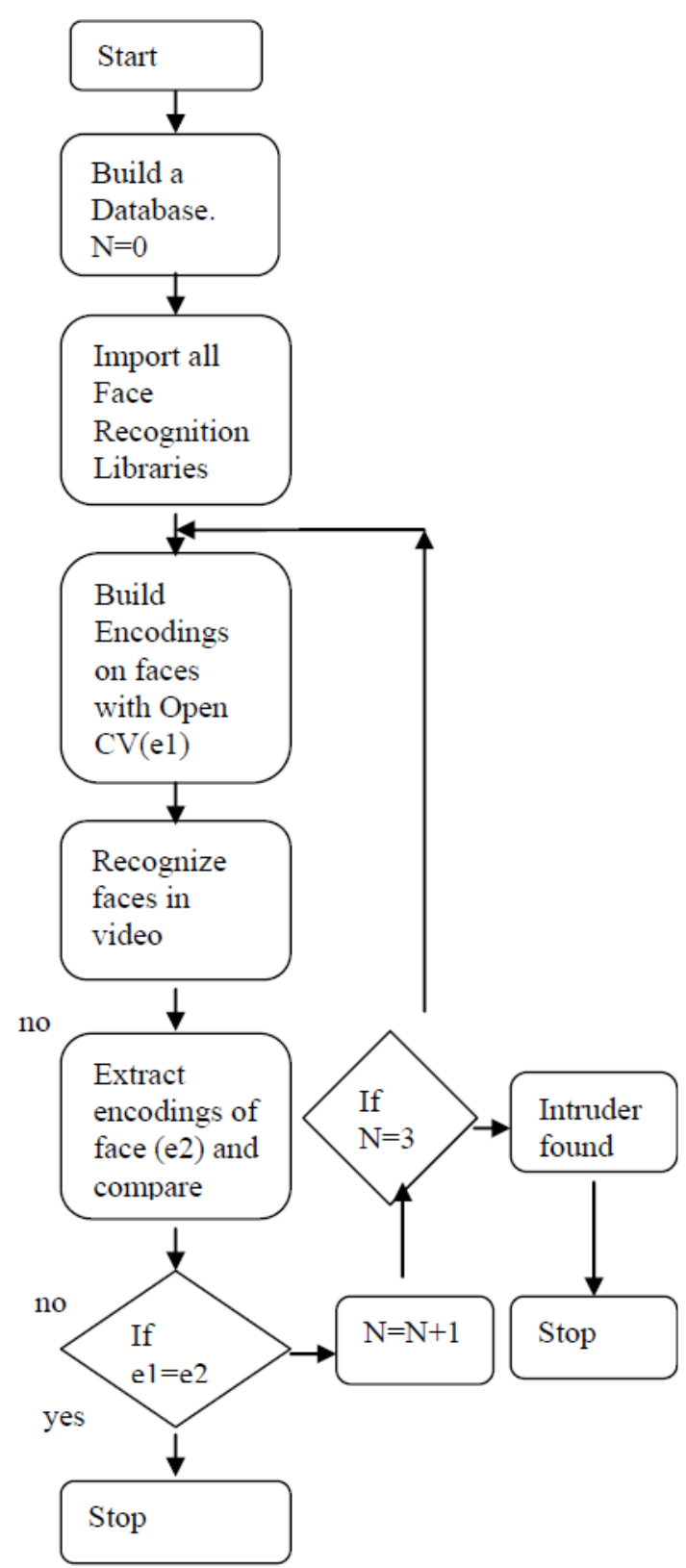

Fig 4: Python Flow Chart

In the very beginning of the process of Open CV method, a database of images is been given to the system. These images are of the persons who live in that particular home or office or shop etc. Now, using Open CV method encodings are created on the faces present in the database. Then the video starts recording and detects particular face excluding the pets.

Encodings are created on the face of a person present in the video. Now these encodings are compared to the encodings of pictures present in database. If both the encodings match, then he is not an intruder, otherwise it again goes back and compare with encodingson another image in database. This process continues till the database is completely compared. Even though the face is not getting matched, then the person is an intruder. The whole process of creating encodings of face is done in python language.

\section{RESULTS}

1. Overall Project Kit:-

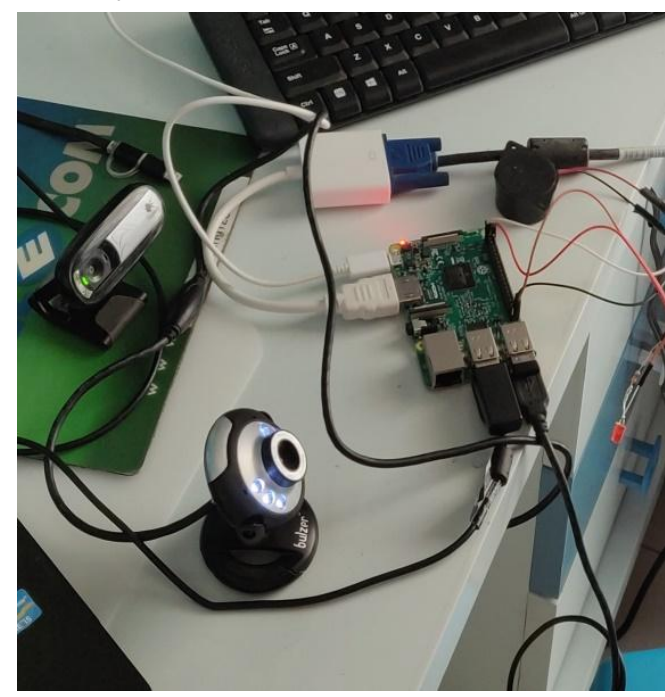

Fig 5:Complete Kit

The overall Project of Iot based Human Intruder Detection System using LabVIEW uses Hardware components like Raspberry pi, webcam, HDMI to VGA converter.

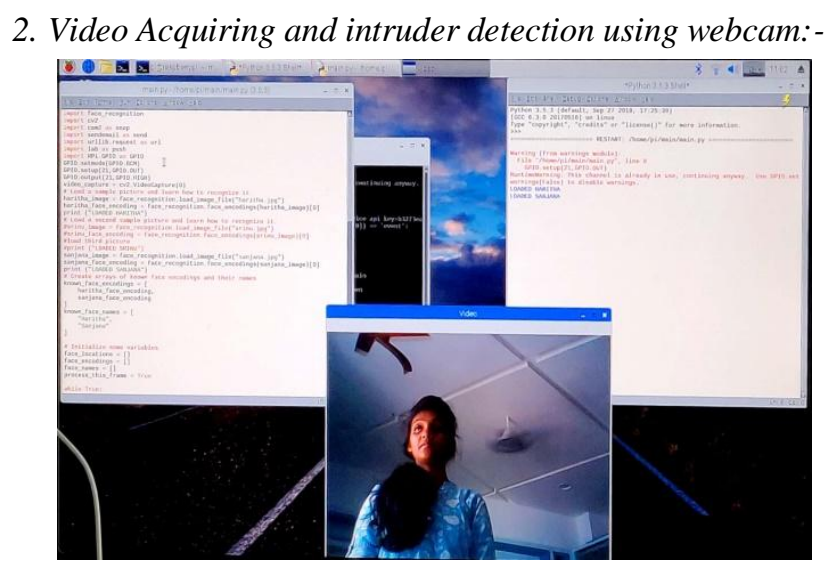

Fig 6: Acquiring video

Once the kit is ready and by providing required commands it starts acquiring the video. Now, it compares the person in the video with the database pictures present in it. Then an email is sent along with the picture of the person.

3. Picture of intruder in email:-

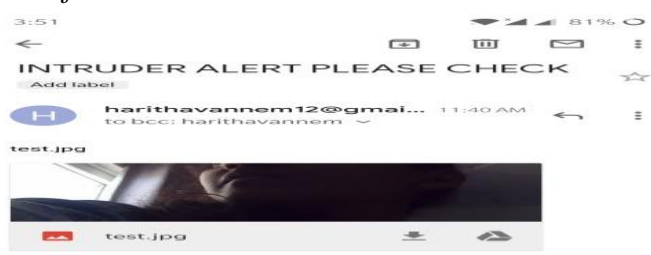

Fig 7: Intruder's picture through email 
After the face comparison process the picture of the intruder is sent through the email for the owner to check italic) in addition to the style provided by the drop down menu to differentiate the head from the text.

\section{APP and Buzzer Control:-}

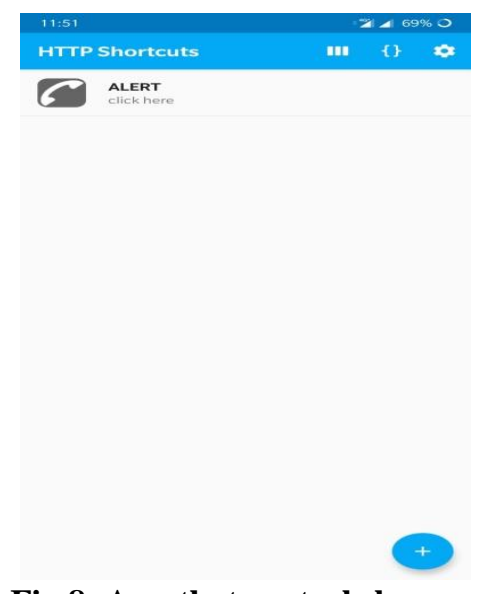

Fig 8: App that controls buzzer

After the email part a notificcation is sent to an application called https shortcuts request through which the owner can control the buzzer .

\section{CONCLUSION}

Generally, these days' people make use of CCTV cameras for security of their home or offices but it has a few disadvantages like, it can't inform to owner about the theft and outside surveillance area it cannot capture evidence or any visual proof. Hence to overcome this disadvantage of existing systems we are implementing this project. In this project we are implementing a raspberry pi based advanced security system programmed with python integrated with LabVIEW to avoid theft. Now with our human intrusion detection system we are able to keep a constant watch over the house. As soon as the system detects any intervention, it provides a real time visual proof with an alert message to the owner. We won't require any memory storage because we are not functioning like a CCTV camera. This system is cost effective and can be used anywhere where security is necessary.

\section{ACKNOWLEDGMENT}

We take opportunity to express our indebt gratitude to the persons who contributed for our work, for being our inspiration and guide which lead to the successful completion of the project.

We express our sincere thanks to K. Rambabu, Assistant Professor, Dept. of ECE, our guide for his valuable suggestion and motivation in successful completion ofproject.

We also wish to express our thanks to all the faculty members, laboratory staff, Electronics and Communication Engineering Department and our college B. V. Raju Institute of Technology who were helpful directly and indirectly for the completion of the project.

\section{REFERENCES}

1. Radke, R.J.; Andra, S.; Al-Kofahi, O.; Roysam, B., "Image change detection algorithms: a systematic survey," Image Processing, IEEE Transactions on, vol.14, no.3, pp.294,307, March 2005.

2. Sampson, R, "False Burglar ". Retrieved April, 2014 Available: http://www.cops.usdoj.gov/pdf/e05021556.pdf.

3. Rose, T, "Change Detection Images". Retrieved April , 2014Available:http://www.tracyleerose.com/Txt/Tutorials/Tut orial_Changedetection/Cha ngedetection.html.

4. "The Raspberry Pi Education Manual," no. 1.0, Dec. 2012.

5. S. Sneha, "IP Camera Video Surveillance using Raspberry Pi.," Feb. 2015.

6. B. Udaya Kumar, D. S. Murty, Ch. R. Phani Kumar, "Implementation of Low Cost Ethernet Based Home Security Using Wireless Sensor Network", Journal published at Algorithms Research, March 2013

7. Lerato Masupha, Tranos Zuva, Seleman Ngwira, Omobayo Esan, "Face Recognition Techniques, their Advantages, Disadvantages and Performance Evaluation", IEEE 2015

8. Paul Viola, Michael J. Jones, "Robust Real-Time Face Detection", International Journal of Computer Vision 57(2), 2004. 